\title{
Development of junction temperature monitoring platform for IGBT charging device adapted to the integration of charging, light and storage
}

\author{
Yu Wenbin ${ }^{1 *}$, Wu Bin ${ }^{2}$, Jia Junguo ${ }^{3}$, and Zhang Feng ${ }^{1}$ \\ ${ }^{1}$ State Grid Smart Energy Transportation Technology Innovation Centre (Suzhou) Co., Ltd., 215010, Suzhou, Jiangsu, China \\ ${ }^{2}$ State Grid Corporation, Production Department, 100032, Beijing, Beijing, China \\ ${ }^{3}$ State Grid Electric Vehicle Service Co., Ltd., 100032, Beijing, Beijing, China
}

\begin{abstract}
With the continuous development of new energy, the power and capacity configuration of charging devices is getting larger and larger, and there are more and more data nodes that charging devices need to manage and monitor. In order to improve the security of the system, it is necessary to monitor the node information of each device in real time. This paper summarizes the data required for high-voltage modelling and junction temperature calculation of high-voltage power electronic devices to develop a monitoring platform for charging devices. The platform records the data of each module in the charging device online, and provides measured data for equipment selection and fault analysis of the charging device. At the end of the article, some experiments were carried out to verify the operation effect of the monitoring platform.
\end{abstract}

\section{Introduction}

The development of "optical storage and charging" integration is one of the important measures to achieve carbon neutrality. The integrated charging station of "optical storage and charging" is a high-tech green charging mode that realizes the coordinated support of photovoltaic, energy storage, and intelligent charging through "storing piles by light". The smart microgrid system intelligently interacts with the public grid according to demand, uses the battery energy storage system to absorb low-valley electricity, and supports fast charging during peak periods, which not only achieves peak-shaving and valley-filling, but also saves power distribution and capacity filling costs, increases the consumption of new energy, and makes up for the lack of continuity of solar power generation. It is a sustainable energy utilization mode ${ }^{[1]}$.

The integrated optical storage and charging microgrid is a bold innovative attempt in the use of renewable energy, energy storage applications and the construction of new energy vehicle charging stations. Recent years, various provinces and cities have successively achieved zero breakthroughs in the "optical storage and charging" project. Among them, including Fujian, Shaanxi, Hubei, etc., many provinces and cities "optical storage and charging" projects have also been successfully put into operation. The "optical storage and charging" integrated charging station landing project has gradually become a scale and is expected to become the mainstream direction of charging infrastructure development.
In the development process of new energy optical storage charging stations, the power and capacity configuration of energy storage power stations is increasing, and energy storage power stations need to manage and monitor more and more data nodes. In order to improve the security of the system, the node information of each device needs to be monitored in real time, and the frequency of data transmission is required to reach the millisecond level or even higher. This requires a monitoring platform to monitor the integrated charging device of light and storage in real time, especially for Insulated Gate Bipolar Transistor (IGBT) junction temperature monitoring.

\section{Overall design of the monitoring platform}

The main functions realized by the monitoring platform are: (1)Status display. The current working conditions of the charging device is displayed, including information such as voltage, current, IGBT junction temperature, and switching status. (2) Store historical data. LabVIEW is used to read and write Structured Query Language (SQL) database. The platform stores each telemetry data every 1 minute, and stores the remote signal displacement information when the remote signal changes. (3)Control the action of the charging device. During the long-term test, it can automatically issue the opening and closing commands of the charging device. It can also be controlled manually. This function is blocked in field application. (4) Disturbance recorder function. After receiving the remote signal displacement signal, the fault

\footnotetext{
*Corresponding author: yuwb@seetic.com
} 
recorder function automatically starts to collect various remote measurements intensively. (5) Failure alarm. When receiving a fault signal, the platform will automatically start the fault recorder and give an audible alarm to remind the testers to pay attention. The overall structure of the monitoring platform is shown in Fig 1.

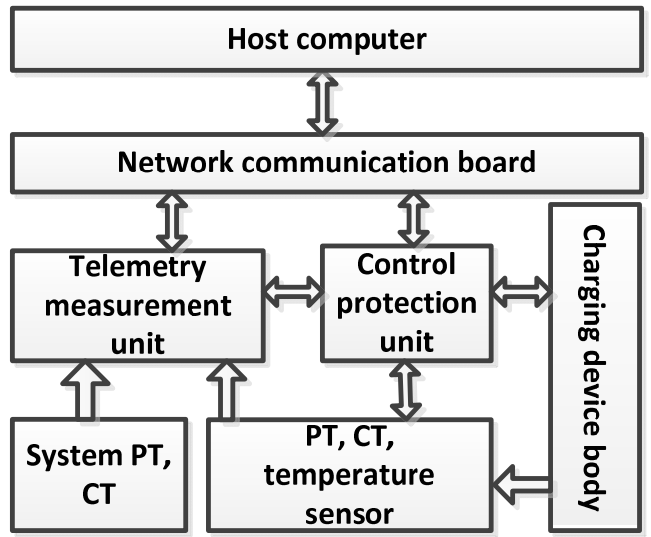

Figure 1. The whole structure chart of monitoring system In order to meet the needs of charging device failure analysis, the platform design focuses on the following aspects: (1)Sampling frequency requirements. The failure model of large-capacity power electronic devices requires the sampling interval of voltage and current to be microseconds ${ }^{[2-3]}$. The sampling frequency of this platform must be able to meet this demand. (2)In order to provide comprehensive data and meet the needs of fault analysis and junction temperature calculation, the monitoring platform designed in this paper needs to collect the terminal voltage of charging device, the current of main branch, transfer branch and arrester, the terminal voltage and current of each IGBT module, the air temperature and the inlet water temperature of each IGBT radiator. (3)Communication rate requirements. Due to the high data sampling frequency during the fault recording and the large number of channels collected by the monitoring platform at the same time, the platform requires a higher communication rate when transmitting the fault recording data.

\section{The hardware design of the monitoring platform}

The hardware design of the monitoring platform mainly includes the selection and design of the host computer, network communication board, telemetry unit, temperature sensor and Potential Tomography (PT) and Computed Tomography (CT). The control and protection unit in Fig 1 can realize the functions of uploading remote signaling data and issuing control commands.

The upper computer adopts industrial computer. In order to meet the communication requirements, the upper computer requires a gigabit Ethernet card. In order to meet the requirements of data storage capacity, the upper computer is equipped with a hard disk of more than $500 \mathrm{G}$.

The main function of the network communication board is the communication interface and protocol conversion to realize the communication between the telemetry unit, the control and protection unit and the upper computer. At the same time, a 128Mbit flash memory is installed on the board to temporarily store the recorded wave data. The network communication board requires the communication rate to meet the requirements, and is stable and reliable; this board is designed with 1 Ethernet communication interface, 5 multimode optical fiber communication interfaces and 4 RS485 serial communication interfaces.

The telemetry unit mainly includes two contents: electrical quantity telemetry and non-electric quantity telemetry:

(1) Electrical quantity measurement. The system voltage and the current flowing through the charging device are collected and calculated using the secondary windings of the on-site PT and CT, and the measurement results of other systems on the site can also be received. In order to meet the requirements of fault analysis, the platform simultaneously collected the current in the main branch, transfer branch and arrester, and collected the voltage across the charging device. In order to meet the functional requirements, PT is installed at both ends of each full-bridge sub-module on this platform; at the same time, PT and CT are installed on \#1 and \#4 IGBTs in the sub-modules. In order to simplify the wiring, the PT and CT secondary measurements of each full-bridge submodule are connected to the central control board of the corresponding module during the design, instead of the telemetry measurement module. In order to meet the needs of IGBT modeling, this platform uses a high-speed AD chip with a sampling frequency of $400 \mathrm{kHz}$ for electrical data collection.

(2) For temperature collection, this platform uses a temperature sensor-AD sampling structure for temperature measurement. In order to calculate the junction temperature of the IGBT, the monitoring platform measures the inlet water temperature and air temperature of each IGBT. Because high-frequency junction temperature is not required for IGBT analysis and calculation, the temperature acquisition of this platform uses an ordinary AD converter, and the actual sampling frequency is $100 \mathrm{~Hz}$. The temperature sensor chooses a smaller contact type thermocouple temperature sensor. For concise wiring, the secondary side of the temperature sensor that measures the water temperature is connected to the central control board of the full bridge sub-module.

\section{Software algorithm of the monitoring platform}

The software design of the monitoring platform includes the software design of the upper computer, the network communication board, the telemetry measurement unit, the control protection unit and the control board of the full bridge sub-module. The upper computer realizes the majority logic calculation function; the network communication board contains the interface protocol conversion program and the recording data storage program; the remote measurement unit and the control board of the full bridge sub-module complete the AD sampling and data communication process; the control 
and protection unit contain a program for uploading remote signaling data.

\subsection{Main program structure and algorithm}

The upper computer adopts LabVIEW programming of NI Company to realize. The main functions of the upper computer software are: (1) Database management function. This platform uses LabVIEW to read SQL data. (2)Data collection calculation and data display function. (3) Disturbance recorder and recorder data reading. (4) Junction temperature calculation function. (5)Fault alarm function. (6) Remote control command issuing function.

Part of the flow chart of the main program is shown in Fig 2.

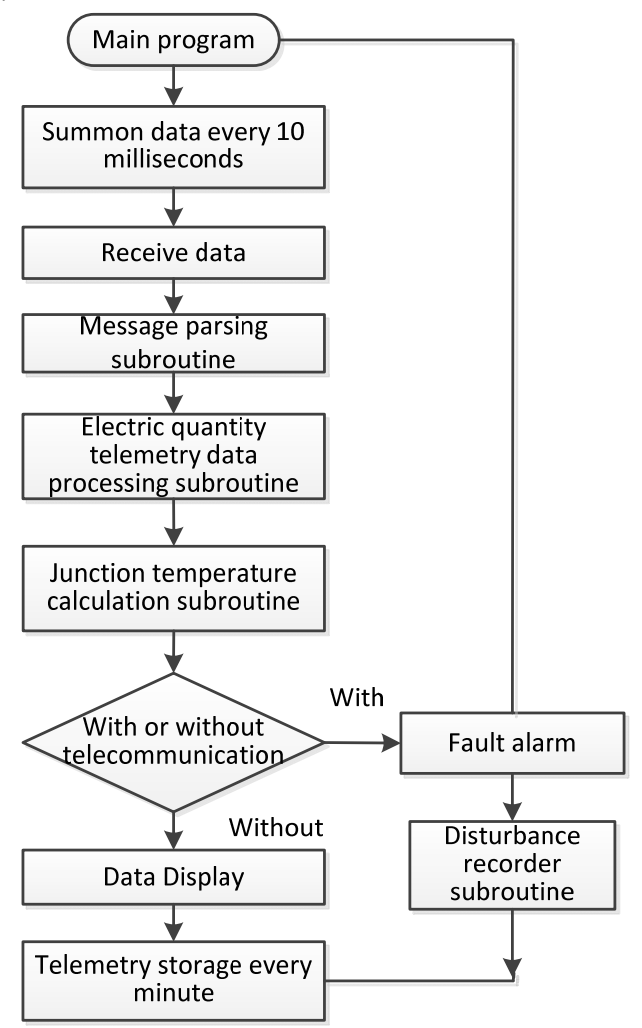

Figure 2. The flow chart of upper-computer main program

\subsection{Voltage and current measurement algorithm}

Voltage and current measurement and data processing are completed in the control board of the full bridge submodule, the telemetry measurement unit, the network communication board and the upper computer. Among them, the central control board and the telemetry measurement unit complete the collection of raw data; the network communication board completes the interface protocol conversion and transmits the data to the upper computer; the upper computer completes functions such as data processing, storage and display. In order to measure the relevant data of each IGBT, the platform collects the voltage at both ends of the full bridge sub module, and simultaneously measures the voltage and current of \# 1 and \# 4 IGBT terminals in each sub module. The relevant data of \# 2 and \# 3 IGBT are obtained through calculation.
According to different design methods, the voltage measurement of HVDC power transmission system has its own methods, and the basic principle is to use resistance or resistance-capacitance voltage division. The voltage measurement of this platform adopts the resistance voltage divider, this platform uses 16-bit AD.

This platform uses optical CT for current measurement of HVDC transmission system. The secondary side of the CT is an optical signal, and the corresponding electronic processing programs are integrated on the secondary side of the $\mathrm{CT}$, and the measurement results are directly output to other devices. Therefore, the platform retains the communication interface to receive the secondary measurement results transmitted by the optical CT.

\subsection{Junction temperature measurement and calculation}

This platform uses the RC thermal network method based on the Causer network model to calculate the junction temperature of the IGBT online. The calculation result of the junction temperature can be used for fault analysis of the IGBT module, as well as for the selection of the IGBT heat $\operatorname{sink}^{[4-5]}$.

\subsubsection{Calculation of steady-state junction temperature}

The calculation formula for the steady-state junction temperature of the IGBT module is as follows:

$$
T_{\mathrm{j}}=\left(R_{j c}+R_{c s}+R_{s a}\right) P+T_{i n}
$$

In the formula: $\mathrm{T}_{\mathrm{j}}$ - The highest operating temperature of IGBT, in $\mathrm{K}$, generally requires less than $125^{\circ} \mathrm{C}$; $\mathrm{R}_{\mathrm{jc}}$ Junction-to-case thermal resistance, which is related to the manufacturing process of IGBT, and is an important technical indicator of IGBT. Manufacturers have regulations, and its value is not affected by the selected radiator or other controlled parameters; $\mathrm{R}_{\mathrm{cs}}$ - contact thermal resistance; $\mathrm{R}_{\mathrm{sa}}$ - thermal resistance of the radiator, which can be obtained according to the thermal resistance curve of the radiator; tin- water temperature, in $\mathrm{K}$; $\mathrm{P}$ component consumption, in $\mathrm{W}$. The above calculation method is also applicable to diodes.

\subsubsection{Calculation of transient junction temperature}

The core algorithm of the foster network model is to use the 3 to 4 order time exponential function to fit the transient thermal impedance curve of IGBT and diode. The fitting relationship is shown in Equation $2^{[6]}$.

$$
Z_{t}=\sum R_{i}\left(1-e^{-t / \tau_{i}}\right)
$$

In the formula: $Z_{t}$ is the junction temperature rise per unit power consumption, in $\mathrm{K} / \mathrm{kW}, \mathrm{Ri}$ is the fitting impedance, $\tau_{\mathrm{i}}$ is the fitting time constant, and $\mathrm{t}$ is the current duration.

According to the physical structure of the IGBT, each layer of the IGBT is simply regarded as a T-shaped connection of thermal resistance and thermal capacity. In this way, the thermal resistance and thermal capacity of 
each layer of thermally conductive objects are connected in cascade to obtain a network model of the thermal impedance of the IGBT.

Because this IGBT carries a heat sink, in order to be able to calculate the junction temperature conveniently, the Causer network model is calculated on the basis of the foster network. The calculation process is realized by computer programming.

\subsubsection{Calculation of transient junction temperature with heat sink}

In order to obtain the IGBT thermal impedance model with a heat sink, it is necessary to fit the thermal resistance curve of the heat sink. The calculation method is as follows:

The exponential function polynomial is used to fit the transient thermal resistance curve of the IGBT junction to obtain the $\mathrm{R}$ and $\mathrm{C}$ parameters in the foster model. The fitting formula is:

$$
\begin{gathered}
Z_{j c}=\sum_{i} Z_{i}=\sum_{i} R_{i}\left(1-e^{-t / \tau_{i}}\right)=\sum_{i} R_{i}\left(1-e^{-t / R_{i} C_{i}}\right) \\
\tau_{i}=R_{i} C_{i}
\end{gathered}
$$

$\mathrm{Ri}$ and $\tau_{\mathrm{i}}$ in the fitting formula correspond to Ri and $\tau_{\mathrm{i}}$ in the network foster model.

After the fitting is completed, the thermal impedance model of the IGBT with heat sink is obtained as shown in Fig. 3.

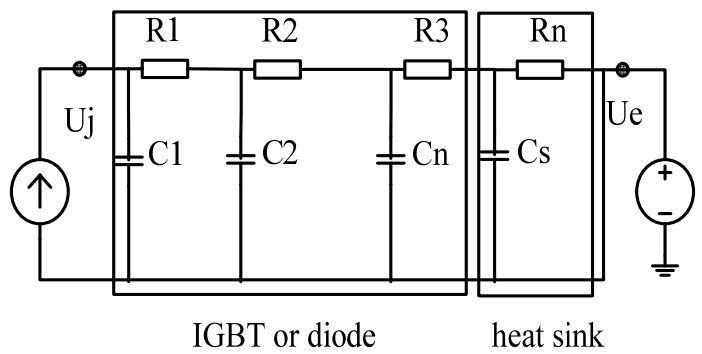

Figure 3. Causer network of thyristor including heatsink

Before calculation, it is necessary to judge whether the terminal voltage applied to the IGBT is forward or reverse according to the terminal voltage of the full-bridge submodule. If the full-bridge sub-module terminal voltage is positive (the voltage on the left is higher than the right), the \#2 and \#4 IGBTs in the sub-module will flow, and the diodes of \#1 and \#3 will flow. On the contrary, \#2 and \#4 diodes pass current, and \#1 and \#3 IGBTs pass current. According to the current flow, it is decided whether to use the related parameters of the IGBT or the diode related parameters.

\subsection{Disturbance recorder subroutine flow}

The processing method of the fault recorder data is different from the processing method of the normal data, which requires a large amount of data storage, high storage speed, and easy copy transfer. In the implementation, LabVIEW does not adopt the form of reading and writing databases, but adopts a more concise way of reading and writing files in LabVIEW. Disturbance recorder requires the following functions: 1) Any remote signal change must start the fault recorder program, including fault alarm signals and switch action signals. If the fault signal sent is the same as the last time, the fault recorder will not be started. 2) There are two operation modes: manual start and automatic start. Automatic recording is to automatically start recording when there is a remote signal change, while manual recording is to send a recording command on the upper computer to record. 3) It has the function of reading fault data, which is convenient for analysis and calculation. 4) The recording content is a variety of measurement signals in the time range from $0.5 \mathrm{~s}$ before the start of the recording command to $1.5 \mathrm{~s}$ after the start of the recording command. The short-circuit fault of HV and UHV DC generally lasts for a few milliseconds. Considering the time required for signal transmission and fault judgment, $0.5 \mathrm{~s}$ before the start of the recording command can meet the recording requirements. Due to the need to record the action of the charging device after a grid failure, considering special circumstances such as circuit breaker refusal, delay, and failure, the platform fault recording continues until $1.5 \mathrm{~s}$ after the start of the recording command.

In order to meet the needs of modeling and analysis in the future, the voltage and current signals of the fault recording are sampled once at $2.5 \mu \mathrm{s}$, that is, the sampling frequency is $400 \mathrm{kHz}$; the sampling frequency of junction temperature is not too high. The sampling frequency of this platform is $100 \mathrm{~Hz}$.

Because of the large amount of communication data of the fault recording, in order to relieve the communication pressure in a short time, the fault recording program is divided into two parts: 1) First, store the recording data in the network communication board. After the recording is completed, delete the earliest recording data in the communication board, and then send the command to collect the recording data to the upper computer. The network communication board stores the most recent ten wave recording data. 2) After the host computer receives the instruction to collect the oscillography data, it starts to collect the oscillography data, and the host computer stores all the oscillography data. The host computer stores the collected signals in an excel file, and the file is named according to the time when the wave actually started recording. The fault record data and the normal data use different port numbers to communicate with the upper computer. The recording workflow is shown in Fig 4. The host computer uses the normal communication channel to send the fault recording command at the beginning of the recording. After receiving the fault recording in the network communication board, it opens the fault recording channel, sends the upload recording data command, and transmits the recording data. 


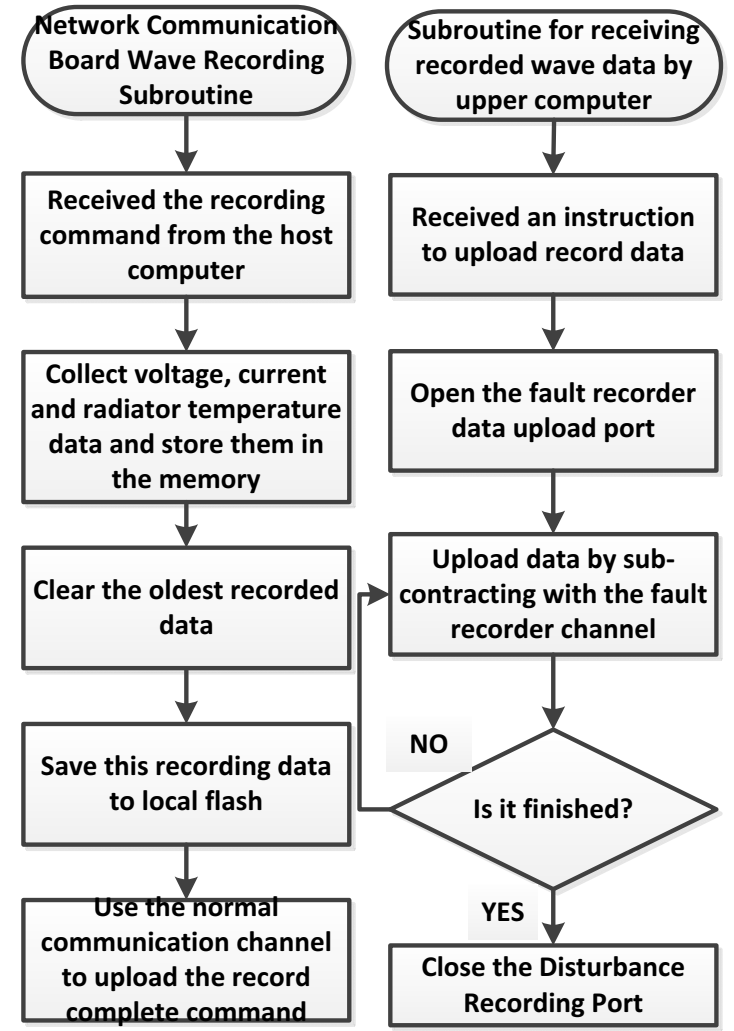

Figure 4. Data recording program flow chart

\section{Experimental verifications}

Through the monitoring platform, the charging device has been monitored for a long time, and various functions such as command issuance, fault recording, status display, voltage and current, and junction temperature data acquisition and storage have been completed. The monitoring platform has played an important role in various tests such as the current flow test and insulation test of the whole school sub-modules of the charging device.

1) Verification of junction temperature calculation results.

This experiment uses the method of comparing the simulation calculation results of the same experiment with the results of this platform to verify. The relevant parameters of the radiator in this article are as follows: $\mathrm{R}=0.0043 \mathrm{~K} / \mathrm{W}$; thermal contact resistance: $0.009 \mathrm{~K} / \mathrm{W}$; $\mathrm{C}=\mathrm{mCp}=3048 \mathrm{~J} / \mathrm{s} \bullet \mathrm{K}$; water inlet temperature: $40^{\circ} \mathrm{C}$. IGBT adopts 52NA $2000 \mathrm{~K} 451300$ of ABB.

Table1. The simulation calculation results of main branch \#1 sub module junction temperature

\begin{tabular}{|c|c|}
\hline Calculation item & $\# 1$ full bridge sub-module \\
(unit ${ }^{\circ} \mathrm{C}$ )
\end{tabular}

When the full-bridge sub-module is forwardconducting, there is no current in the \#1 and \#3 IGBT modules, and the current flows through the \#1 and \#3 IGBT parallel diodes and the \#2 and \#4 IGBT bodies. The method in literature is used to simulate and calculate the maximum junction temperature of the \#1 and \#3 IGBT parallel diodes and \#2 and \#4 IGBT bodies in the main branch \#1 full bridge submodule. The results are shown in Table 1. The results of the highest junction temperature recorded by the test platform are shown in Table 2 . From the analysis of the calculation results, the junction temperature calculation results of this platform are similar to the offline junction temperature calculation results, which can correctly reflect the actual junction temperature. Table2. Main branch \#1 full bridge sub-module monitoring platform junction temperature calculation results

\begin{tabular}{|c|c|}
\hline Calculation item & \#1 full bridge sub-module \\
(unit ${ }^{\circ} \mathrm{C}$ )
\end{tabular}

2) Disturbance record data result verification

In order to verify the function of fault recording and broadcasting data, a monitoring platform and an oscilloscope are used in the disconnection test of the charging device to conduct a monitoring data comparison test. Through the data collected in the two ways, it is found that the curves of the two are roughly the same, which can clearly reflect the key information in the fault recorder, which also shows that the wave recorder function of the designed monitoring platform meets the measurement requirements.

\section{Conclusion}

This paper designs a junction temperature monitoring platform for IGBT charging devices that adapts to the integration of charging, light and storage, and draws the following conclusions:

(1) The monitoring platform designed in this paper uses high sampling frequency for data fault recording, which provides effective measured data for high-voltage modeling and fault analysis of large-capacity power electronic equipment.

(2) Through experimental verification, the monitoring platform has realized the online junction temperature measurement and calculation of power electronic equipment, which provides actual measurement basis for the verification of the safe working area of the IGBT and the selection of the radiator. 


\section{References}

1. Jiang Xiaobei. (2021). "Optical storage and charging" integrated microgrid will usher in great development. New Energy Technology (02), 33-34+36. doi:CNKI:SUN:JSQG.0.2021-02-020 .

2. Zhao Zhengming, He Fanbo, Yuan Liqiang. Electromagnetic transient analysis technology and application of large-capacity power electronic system. Proceedings of the Chinese Society for Electrical Engineering, 2014, 34(18): 3013-3019

3. Ji Shiqi, Zhao Zhengming, Yuan Liqiang. Analysis of transient mechanism model of high voltage IGBT. Journal of Tsinghua University. 2012.52(11):15781583

4. FELLER L, HARTMANN S, SCHNEIDER D. Lifetime analysis of solder joints in high power IGBT modules for increasing there liability for operation at $150 \%[\mathrm{~J}]$. Microelectronics Reliability, 2008, 48(9): 1161-1166.

5. LU H, BAILEY C, YIN C. Design for reliability of power electronics modules[J]. Microelectronics Reliability, 2009, 49(9 / 10 / 11): 1250-1255.

6. Luo Z, Ahn H. A thermal model for insulated gate bipolar transistor module[J] . IEEE Transactions on Power Electronics, 2004, 19(4) : 902-907. 